\title{
Zygosity Diagnosis in Young Twins by Questionnaire for Twins' Mothers and Twins' Self-reports
}

\author{
Syuichi Ooki' and Akio Asaka ${ }^{2}$ \\ ' Department of Clinical Bioinformatics, Graduate School of Medicine, the University of Tokyo, Tokyo, Japan \\ ${ }^{2}$ Joto Hospital,Yamanashi, Japan
}

$T^{\text {ne }}$ he present study deals with the determination of zygosity in twins of childhood age by simple questionnaire. The subjects were 224 twin pairs and their mothers, consisting of 159 monozygotic and 65 same-sex dizygotic pairs, identified by genetic markers including DNA samples. Mothers of twins responded to 19 questionnaire items dealing with twin similarity in 16 items about physical features and 3 items about the degree of similarity and frequency of being mistaken (confusion of identity) when twins were about 1 year of age. The twins themselves responded to three questionnaire items dealing with only confusion of identity items. The results of stepwise logistic regression analysis were as follows: the total accuracy of the mothers' questionnaire was $91.5 \%$ when using only the items dealing with confusion of identity. This accuracy was slightly lower than that obtained by twins' selfreports dealing with nearly the same question items of confusion of identity, answered by both twins separately with $93.3 \%$ accuracy. The total accuracy of mothers' questionnaire responses rose to $95.1 \%$ when we used all 19 items. In addition to "the frequency of being mistaken", two physical features, namely "shape of fingers" and "shape of eyebrow", were very informative. In conclusion, twin zygosity can be estimated by the use of the mothers' simple questionnaire with sufficient accuracy even in very young twins about 1 year of age.

More than 20 studies have shown that the determination of zygosity in twins based on questionnaires can be done with considerable accuracy. These studies are summarized by a recent study of Rietveld et al. (2000) showing that the accuracy of the questionnaires employed is around 95\%. The usefulness of parental reports has been gradually recognized (Bønnelykke et al., 1989; Chen et al., 1999; Cohen et al., 1973, 1975; Price et al., 2000; Rietveld et al., 2000).

We developed a zygosity questionnaire for twins themselves (Ooki et al., 1990) and for twins' mothers (Ooki et al., 1993), mainly for use in a genetic epidemiologic twin study. In Japan, multiple birth rates have been increasing since 1975, and the higher twinning rates since 1987 have been partially attributed to the higher proportion of mothers treated with ovulation-inducing hormones and partially attributed to in-vitro fertilization (Imaizumi \& Nonaka, 1997). The need for an appropriate method of determining zygosity for use by twins' parents or nursing staff has rapidly increased for many reasons (Derom et al., 2001) and is especially important when twins are too young to respond to questions. The purpose of this study was to evaluate the accuracy of the zygosity questionnaire for twins' mothers already used in Japan, by adding questions to collect information about physical similarity, and then to compare the accuracy of the mothers' reports with that of selfreports using the same young twin pairs. although the use of questionnaires for young twins has increased recently (Bønnelykke et al., 1989; Cohen et al., 1973, 1975), the comparison of mothers' reports with that of self-reports is relatively limited (Chen et al., 1999; Ooki et al., 1993).

\section{Materials and Methods \\ Subjects}

As there is no population-based twin registry in Japan, it is very difficult to collect twins' blood samples and mothers' zygosity questionnaires. The original dataset was 74 pairs, consisting of 61 monozygotic (MZ) and 13 same-sex dizygotic (DZ) pairs (Ooki et al., 1993). In this study two pairs (one $\mathrm{MZ}$ and one DZ pair) were removed from the original dataset because of insufficient data. The subsequent dataset consisted of 152 pairs, $99 \mathrm{MZ}$ and 53 DZ pairs. Therefore, the total subjects of this study consisted of $159 \mathrm{MZ}$ and $65 \mathrm{DZ}$ twin pairs and their mothers.

Address for correspondence: Syuichi Ooki, Department of Clinical Bioinformatics, Graduate School of Medicine, the University of Tokyo, 7-3-1 Hongo Bunkyo-ku, Tokyo 113-8655, Japan. Email: sooki@kj8.so-net.ne.jp 
The twins were all applicants and students of the secondary education school attached to the Faculty of Education of the University of Tokyo from 1985 to 2003. This school was established in 1948 and adopted a unique entrance system. About 50 pairs of 12 -year-old twins who live in the Tokyo metropolitan area take an examination every year, and about 15 pairs are admitted. The enrolled twins participate in the twin study of education and related projects. All of the twins' parents must hand in a Twins Protocol Questionnaire, which gathers information on family structure, obstetrical findings of mothers, physical, motor and mental development of twins from birth to 12 years of age, and zygosity. One of the parents of each applicant, usually the mother, participates in a medical interview by two or three interviewers (at least one of the authors being an interviewer in the periods of data collection), where the Twins Protocol Questionnaire is checked carefully to be sure there are no unanswered questions. Questions concerning zygosity determination based on DNA/blood testing are also asked at the medical interview. It was confirmed that most subjects of the present study did not have much knowledge of their zygosity. First, we ascertained dizygotic twin pairs from applicants whose $\mathrm{ABO}$ blood type was discordant using information on obstetrical data described in the "Maternal and Child Health Handbook", presented by the Ministry of Health, Labour and Welfare for all pregnant women who are registered. This handbook was also used in the medical interview. A more accurate zygosity diagnosis using many genetic markers or DNA polymorphisms is performed for those twinpairs who are admitted to the school.

\section{Zygosity Questionnaire}

Our zygosity questionnaire for twins themselves (Ooki et al., 1990) and for twins' mothers (Ooki et al., 1993) asked about confusion of identity between twins (see Appendix A and Appendix B). The questionnaire for twins' mothers is shown in Appendix A. The questionnaires were divided into two parts. The first part contained 16 items regarding mainly physical similarities at about 1 year of age. According to the answers, the following similarity points were given: 1 (no difference), 2 (do not know), and 3 (clear difference). These items have not previously been used for zygosity classification. The three questions of the second part asked mothers whether the twins were "like two peas in a pod" and whether they were "mistaken for one another and by whom" at about 1 year of age. The usefulness of these questions has already been reported (Ooki et al., 1993), and this questionnaire has been used mainly for childhood-age twins in Japan. According to the degree of similarity, 1 to 3 or 1 to 4 points were allotted, and then the points were totaled, with distribution from 3 (1,1,1 for each answer) to 10 (3,3,4 for each answer). Zygosity was determined according to a cut-off point on the single summed raw score. Torgersen (1979) used this method originally in a questionnaire for twin pairs, and our questionnaire for mothers was derived from Torgersen's questionnaire. If the summed score was 3-6, twin pairs were classified as monozygotic, and if the sum was 7-10, they were termed dizygotic. The determination of the cut-off point can be flexible according to the particular use of the data. This method has relatively high accuracy, around $90 \%$. The problem of this questionnaire is the narrow range of the summed score. The possibility of using information on physical features had been considered as a way to resolve this weak point.

Because the twins in this study were around 12 years of age, their similarity at about one year, which was explored in the questionnaire, had occurred 10 or more years ago, so answers based on mothers' memories might not be accurate. If mothers could not remember their children's similarity, the interviewer recommended that they choose the answer "do not know" or make an intuitive answer.

The questionnaire for twins is shown in Appendix B. Each twin answered the same questionnaire separately. The questionnaire included three questions regarding similarity and confusion: "How are you alike?" "How often are you mistaken for your twin?" and "By whom are you mistaken for your twin?" According to the degree of similarity reported, 1-3, 13 , and 1-4 points were allotted, respectively, for these three questions, and then the sum of the points for both twins was calculated. This sum could range from 6 (1,1,1 for each answer for each twin) to $20(3,3,4$ for each answer for each twin). Zygosity was determined according to the given cut-off point on the single summed raw score. Torgersen (1979) developed this method originally, and a translated version was used in Japan for twins of a wide range of ages. Determination of the cut-off point can be flexible according to the particular use of the data.

\section{Zygosity Testing}

Zygosity determination of the subjects who are admitted to the school was performed using many genetic markers, which were slightly different according to the entrance year. The zygosity testing included $\mathrm{ABO}, \mathrm{CcDEe}, \mathrm{MNSs}$, Haptoglobin, Acid phosphatase, Glutamate pyruvate transaminase, Estrase D, HLA-DR, DNA polymorphisms (for example, betaglobin gene cluster haplotype, Dopamine receptor gene: DRD4, Serotonine receptor gene: 5-HTT, and mtDNA 9bp deletion), and related tests by blood sample, and DNase2 by urea sample. As a rule twins and their parents were examined. If all markers are concordant for certain twin pairs, the probability of being $\mathrm{MZ}$ calculated using Bayes theorem is more than 0.999 .

\section{Statistical Procedures}

First, we analyzed the usefulness of physical features for determining zygosity. As shown in Appendix C, three indexes were determined. Index A is the percentage of 
time that mothers of MZ twins answered "no difference" to a certain item to which mothers of DZ twins answered "clear difference". Index B is the percentage of time that mothers of MZ twins answered "clear difference" to a certain item to which mothers of DZ twins answered "no difference". Index $C$ is the percentage of time that mothers of both $\mathrm{MZ}$ and $\mathrm{DZ}$ twins answered "do not know" to a certain item. By definition, the sum of Index A, Index B, and Index C is equal to $100 \%$ for each item. Obviously items that have a high value for Index A and low values for Index B and Index C were very informative.

Next, stepwise logistic regression analyses were performed on the six patterns of selected question items to compare their accuracy. We used the PROC LOGISTIC program (SAS Institute, 1993) with a significance level of .10 for entry into or retention in the model. The total accuracy, sensitivity and specificity of the questionnaire at specific cut-off points were calculated by specifying "CTABLE" options. Here sensitivity means DZ pairs classified correctly as DZ and specificity means $M Z$ pairs classified correctly as $M Z$. In the analysis of twins' self-reports, twins were analyzed as part of a pair or as individuals. In the former case, summed points of both twins for each three questions were used as independent variables.

Finally, we analyzed the usefulness of summed score method for selected items. The accuracy of the original dataset (Ooki et al., 1993) was also compared to that of the subsequent dataset.

\section{$\overline{\text { Results }}$}

Index A, Index B, and Index C of the sample are summarized in Table 1. "Shape of eyebrow", "shape of fingers", and "sleeping face" seemed to be useful, with Index A $>75 \%$, Index B $<10 \%$, and Index C $<20 \%$.

The results of logistic regression analysis are summarized in Table 2. As seen in Table 2, total accuracy was $91.5 \%$ when we used the 16 items in the mothers' questionnaires dealing with physical similarity (first part of the mothers' questionnaire) (Pattern 1). The total accuracy was $91.5 \%$ when we used the three items dealing with confusion of identity (second part of the mothers' questionnaire) (Pattern 2). The total accuracy was $95.1 \%$, with a sensitivity of $92.3 \%$ and a specificity of $96.2 \%$, when we used all 19 items (Pattern 3). In addition to "the frequency of being mistaken for one another $\left(I M_{18}\right)$ ", "shape of fingers $\left(I M_{11}\right)$ ", and "shape of eyebrow $\left(I M_{4}\right)$ " were selected with a significance level of .10 . The total accuracy was $93.3 \%$ when using twins' self-reports (Pattern 4). This accuracy was nearly the same, even if self-reports of only one member of the twin pair were analyzed (comparison between Pattern 4' and Pattern 4"). The total accuracy was not changed very much if both mothers' reports and twins' self-reports were analyzed simultaneously (Pattern 5 or Pattern 6). Sensitivity was much lower than specificity when using the items dealing with only confusion of identity (Pattern 2, Pattern 4, or Pattern 5).

The distributions of summed scores for selected items according to determined zygosity are shown in Table 3. The mothers' three items and the twins' three items were the same as items we previously reported (Ooki et al., 1990, 1993). The difference of distribution of summed scores between MZ and DZ pairs was clearly seen. With the mothers' three items, if the cut-off point was set between the score 6 and 7

Table 1

The Results of Similarity of Physical Features Reported by Twins' Mothers

\begin{tabular}{lccc}
\hline & Index A & Index B & Index C \\
\hline 1. facial appearance & $61.6 \%$ & $8.5 \%$ & $29.9 \%$ \\
2. the number of the hair whorl & $59.4 \%$ & $19.2 \%$ & $21.4 \%$ \\
3. the position of the hair whorl & $52.2 \%$ & $17.9 \%$ & $29.9 \%$ \\
4. shape of eyebrow & $76.3 \%$ & $7.6 \%$ & $16.1 \%$ \\
5. shape of eyelid & $61.2 \%$ & $11.6 \%$ & $27.2 \%$ \\
6. shape of eye & $58.9 \%$ & $12.5 \%$ & $28.6 \%$ \\
7. shape of ear & $62.9 \%$ & $8.5 \%$ & $28.6 \%$ \\
8. voice & $52.7 \%$ & $11.6 \%$ & $35.7 \%$ \\
9. the number of the mole or spot & $26.3 \%$ & $26.8 \%$ & $46.9 \%$ \\
10. the position of the mole or spot & $28.1 \%$ & $33.5 \%$ & $38.4 \%$ \\
11. shape of fingers & $75.4 \%$ & $5.4 \%$ & $19.2 \%$ \\
12. physique & $71.9 \%$ & $7.6 \%$ & $20.5 \%$ \\
13. sleeping face & $77.2 \%$ & $9.4 \%$ & $13.4 \%$ \\
14. sleeping posture & $65.2 \%$ & $9.8 \%$ & $25.0 \%$ \\
15. liability to get illness & $59.4 \%$ & $12.9 \%$ & $27.7 \%$ \\
16. tendency of concordance for illness & $63.4 \%$ & $18.3 \%$ & $18.3 \%$ \\
\hline
\end{tabular}

Note: See text and Appendix $C$ as to the explanation of each index; Index $A+\operatorname{Index} B+\operatorname{Index} C=100 \% ; n=224$ mothers of twin pairs, consisteing of $159 \mathrm{MZ}$ and $65 \mathrm{DZ}$. 


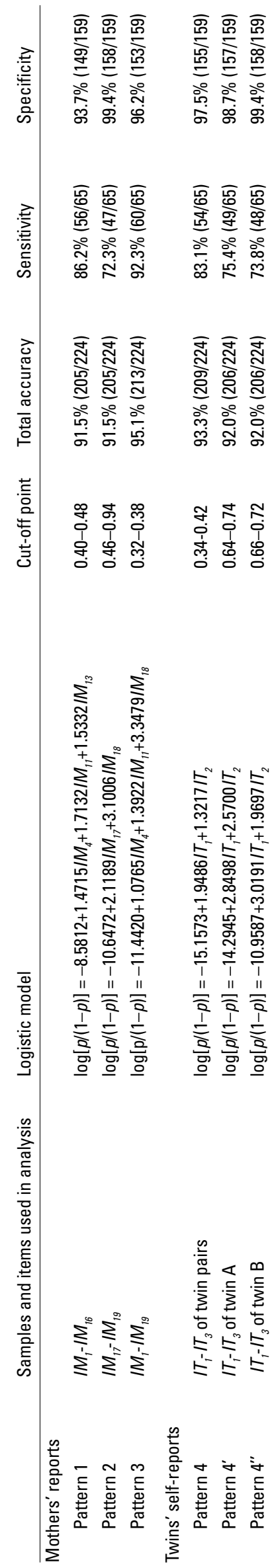

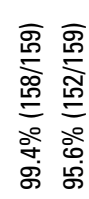

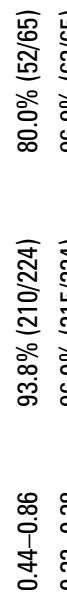

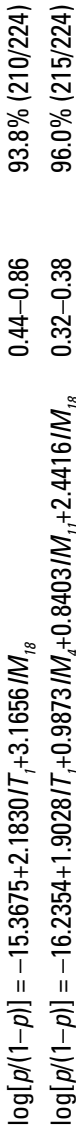

\section{$\overline{\bar{x}}$}

according to our previous study (Ooki et al., 1993), total accuracy was $89.7 \%$ (201/224), with accuracy of $\mathrm{MZ}$ equal to $95.6 \%(152 / 159)$ and that of $\mathrm{DZ}$ equal to $75.4 \%$ (49/65). With the twins' three items, if the cut-off point was set between the score 13 and 14 according to our previous study (Ooki et al., $1990)$, total accuracy was $90.2 \%(202 / 224)$, with accuracy of MZ equal to $89.3 \%(142 / 159)$ and that of DZ equal to $92.3 \%(60 / 65)$. These results were not satisfactory. With the mothers' five items, if the cutoff point was set between the score 9 and 10, total accuracy was $94.6 \%(212 / 224)$, with accuracy of MZ equal to $95.0 \%(151 / 159)$ and that of DZ equal to $93.8 \%(61 / 65)$.

The results of simple summed score method using original three items of mothers' questionnaire according to dataset were shown in Table 4. The deterioration in total accuracy was observed due to the change in composition of the sample.

\section{$\overline{\text { Discussion }}$}

One of the main focuses of this study was to evaluate the already reported zygosity questionnaire used in Japan for childhood-age twins and answered by their mothers (Ooki et al., 1993). In Japan it is said that even now zygosity misclassification based on inappropriate placental findings is very high. Therefore, it is important to offer a simple and proper method to classify zygosity of childhood-age twins. The reason maternal reports were used is that in Japan, in general, mothers bring up their children, including multiples. Some reports suggest the usefulness of information from fathers (Chen et al., 1999; Rietveld et al., 2000), but cultural differences should be considered. The similarity of twin pairs at about 1 year of age was quite informative, which was in accordance with findings of a recent study (Price et al., 2000).

The twin subjects used in this study were all Japanese infants. As a result, some items that were useful for zygosity determination in Caucasian samples, such as eye color, hair color, and facial color (Peeters et al., 1998; Spitz et al., 1996), were not useful because there was less variation. This study suggested that similarity of "shape of fingers" and "shape of eyebrow" during infancy were also useful items for zygosity determination, at least in this ethnic group. Recently, we confirmed this finding using two additional groups of twins' mothers (not published). We thought that mothers regard their children as similar or dissimilar intuitively and the reasons for their judgment are highly variable.

The zygosity questionnaire was developed along two dimensions, the similarity of physical characteristics and the confusion of identity (Rietveld et al., 2000). Our original questionnaire for twins' mothers and twins themselves asked only about the latter dimension. 
Table 3

The Distribution of Summed Score with Cut-off Point According to Zygosity and Selected Items

\begin{tabular}{|c|c|c|c|c|c|c|c|}
\hline & \multirow[t]{2}{*}{ Summed Score } & \multicolumn{2}{|c|}{ Mothers' Three Items } & \multicolumn{2}{|c|}{ Mothers' Five Items } & \multicolumn{2}{|c|}{ Twins' Three Items } \\
\hline & & $\mathrm{MZ}(n=159)$ & $\mathrm{DZ}(n=65)$ & $\mathrm{MZ}(n=159)$ & $\mathrm{DZ}(n=65)$ & $\mathrm{MZ}(n=159)$ & $\mathrm{DZ}(n=65)$ \\
\hline \multirow[t]{17}{*}{ Similar } & 3 & 7 & 0 & & & & \\
\hline & 4 & 93 & 1 & & & & \\
\hline & 5 & 37 & 2 & 7 & 0 & & \\
\hline & 6 & 15 & 13 & 78 & 1 & 4 & 0 \\
\hline & 7 & 6 & 7 & 36 & 0 & 0 & 0 \\
\hline & 8 & 1 & 0 & 16 & 1 & 20 & 0 \\
\hline & 9 & 0 & 13 & 14 & 2 & 19 & 1 \\
\hline & 10 & 0 & 29 & 3 & 5 & 39 & 0 \\
\hline & 11 & & & 4 & 8 & 23 & 1 \\
\hline & 12 & & & 0 & 6 & 22 & 2 \\
\hline & 13 & & & 0 & 7 & 15 & 1 \\
\hline & 14 & & & 1 & 10 & 16 & 9 \\
\hline & 15 & & & 0 & 10 & 1 & 3 \\
\hline & 16 & & & 0 & 15 & 0 & 5 \\
\hline & 17 & & & & & 0 & 1 \\
\hline & 18 & & & & & 0 & 2 \\
\hline & 19 & & & & & 0 & 13 \\
\hline Dissimilar & 20 & & & & & 0 & 27 \\
\hline
\end{tabular}

Note: Mothers' three items are the same as items we previously reported (0oki et al., 1993).

Mothers' five items are the second part of questions (mothers' three items) , "shape of fingers" and "shape of eyebrow".

Twins' three items are the same as items we previously reported (Ooki et al., 1990).

Table 4

The Results of Simple Summed Score Method Using Mothers' Three Items According to Dataset

\begin{tabular}{lcccc}
\hline & MZ:DZ ratio & \multicolumn{1}{c}{ Correct } & DZ classified as DZ & MZ classified as MZ \\
\hline Original dataset $(n=72)$ & $5.00(60 / 12)$ & $94.4 \%(68 / 72)$ & $75.0 \%(9 / 12)$ & $98.3 \%(59 / 60)$ \\
Subsequent dataset $(n=152)$ & $1.87(99 / 53)$ & $88.2 \%(134 / 152)$ & $75.5 \%(40 / 53)$ & $94.9 \%(94 / 99)$ \\
\hline
\end{tabular}

Note: If the summed score was 3-6, twin pairs were classified as MZ, and if the sum was 7-10, they were classified as DZ (0oki et al.,1993).

According to a recent study (Chen et al., 1999) and our previous study (Ooki et al., 1993), either parental reports or self-reports can classify zygosity with nearly the same accuracy in adolescent twins. As shown in Table 2, this tendency was also found in this study, if the similar question items, namely confusion of identity, were used to classify the zygosity. As to self-reports, the accuracy did not decrease even if the reports of only one member of a twin pair were used. The reason seemed to be the high concordance rate of the answers to each question.

Regarding the result of logistic regression analysis, sensitivity was much lower than specificity when we used only items dealing with confusion of identity (Pattern 2 or Pattern 4 in Table 2), which means that the percentage of DZ pairs classified correctly as DZ was much lower than MZ pairs classified correctly as MZ. This tendency has been observed in other studies (Chen et al., 1999; Rietveld et al., 2000). The lower accuracy for $\mathrm{DZ}$ pairs was partly because of the small sample size of $\mathrm{DZ}$ pairs. Another reason for this tendency seemed to be a lack of sensitivity to detecting fraternity in the questionnaire, which asked only about general similarity. Certainly some DZ pairs are so similar in appearance that questions concerning their general similarity cannot always detect their differences.

As shown in Table 3 and Table 4, a simple summed score method using a 3-item measure, which we reported previously (Ooki et al., 1993), was proved to be a less effective method, even if the cutoff point was changed. The misclassification of MZ and DZ, namely specificity and sensitivity, was a trade-off according to the cut-off point.

It is important to know that the accuracy was inflated by not including unclassified twin pairs in the denominator for computing the percentage (Jackson et al., 2001). And total accuracy alone cannot explain the accuracy of "MZ classified as 
$M Z$ " and "DZ classified as DZ", because the ratio of MZ:DZ influences the total accuracy, as shown in Table 4. The total MZ:DZ ratio of this study was 2.45 (159/65). According to Imaizumi (1997), the recent Japanese same-sex MZ:DZ ratio ranges roughly from 2.00 to 3.66 . The MZ:DZ ratio of this study fell within this range.

As shown in Table 2, the total accuracy of the mothers' questionnaire certainly rose to $95.1 \%$ by adding questions dealing with physical similarity, even though the increase in accuracy is not statistically significant. Therefore we thought that the 19-item questionnaire was more practically useful than the 3 -item questionnaire. And in the parsimonious model obtained by logistic regression analysis (Pattern 3), both sensitivity and specificity reached levels greater than $90 \%$, meaning that misclassification ratio is nearly the same between $M Z$ and DZ twin pairs. This is suitable for practical use.

The total accuracy was not changed very much by considering the self-report items dealing with confusion of identity, in addition to mothers' reports (Pattern 6). But, it is relatively easy to obtain both mothers' reports and self-reports for young twins. Therefore, it is worthwhile to collect both types of information if possible. Moreover, the self-report questionnaire could obtain higher accuracy if it included other questions on physical similarity, which have already been gathered in a variety of items for the present young subjects.

As shown in Table 3, the results of simple summed score methods of five items on the mothers' questionnaire showed nearly the same accuracy obtained from logistic regression analysis, suggesting the effectiveness of the simple summed score method in practical use, especially when we use this questionnaire for the purpose of offering zygosity information to twins' mothers more easily. Bønnelykke et al. (1989) stated that it seemed of little use to construct more complex statistical methods, as the summed point of a few simple answers by mothers was sufficient for reliable zygosity classification. Moreover, as Jackson et al. (2001) pointed out, many of the complex formulae for zygosity classification are sample dependent, and accuracy in cross-validation is not always assured. The classification method should vary according to the use to which the questionnaire will be put. The use of a simple sum of checked scores for informative items is both practical and simple.

A limitation of this study is the retrospective methodology of the mothers' reports, which may be informed by factors other than the twins' resemblance as infants. In Japan, where zygosity testing using blood samples cannot be easily or widely performed, answers to the zygosity questionnaire provide very important information. In conclusion, twin zygosity can be estimated by the use of the questionnaire rated by mothers with sufficient accuracy even in very young twins about one year of age. We hope crosscultural adaptation and validation studies (Sumathipala et al., 2000) of this simple questionnaire will be performed in other Asian countries where large twin registers are now under construction.

\section{Acknowledgments}

We would gratefully acknowledge the help of Toshimi Ooma regarding the analysis of the data.

\section{References}

Bønnelykke, B., Hauge, M., Holm, N., Kristoffersen, K., \& Gurtler, H. (1989). Evaluation of zygosity diagnosis in twin pairs below age seven by means of a mailed questionnaire. Acta Geneticae Medicae et Gemellologiae, 38, 305-313.

Chen, W. J., Chang, H. W., Wu, M. Z., Lin, C. C. H., Chang, C., Chiu, Y. N., et al. (1999). Diagnosis of zygosity by questionnaire and polymarker polymerase chain reaction in young twins. Behavior Genetics, 29, 115-123.

Cohen, D. J., Dibble, E., Grawe, J. M., \& Pollin, W. (1973). Separating identical from fraternal twins. Archives of General Psychiatry, 29, 465-469.

Cohen, D. J., Dibble, E., Grawe, J. M., \& Pollin, W. (1975). Reliably separating identical from fraternal twins. Archives of General Psychiatry, 32, 1371-1375.

Derom, R., Bryan, E., Derom, C., Keith, L., \& Vlietinck, R. (2001). Twins, chorionicity and zygosity. Twin Research, 4, 134-136.

Imaizumi, Y., \& Nonaka, K. (1997). The twinning rates by zygosity in Japan, 1975-1994. Acta Geneticae Medicae et Gemellologiae, 46, 9-22.

Jackson, R. W., Snieder, H., Davis, H., \& Treiber, F. A. (2001). Determination of twin zygosity: A comparison of DNA with various questionnaire indices. Twin Research, 4, 12-18.

Ooki, S., Yamada, K., Asaka, A., \& Hayakawa, K. (1990). Zygosity diagnosis of twins by questionnaire. Acta Geneticae Medicae et Gemellologiae, 39, 109-115.

Ooki, S., Yamada, K., \& Asaka, A. (1993). Zygosity diagnosis of twins by questionnaire for twins' mothers. Acta Geneticae Medicae et Gemellologiae, 42, 17-22.

Peeters, H., Van Gestel, S., Vlietinck, R., Derom, C., \& Derom, R. (1998). Validation of a telephone zygosity questionnaire in twins of known zygosity. Behavior Genetics, 28, 159-163.

Price, T. S., Freeman, B., Craig, I., Petrill, S. A., Ebersole, L., \& Plomin, R. (2000). Infant zygosity can be assigned by parental report questionnaire data. Twin Research, 3, 129-133.

Rietveld, M. J. H., van der Valk, J. C., Bongers, I. L., Stroet, T. M., Slagboom, P. E., et al. (2000). Zygosity diagnosis in young twins by parental report. Twin Research, 3, 134-141.

SAS Institute, Inc. (1993). SAS user's guide. Basics and statistics (Version 6.12). Cary, NC: SAS Institute. 
Spitz, E., Moutier, R., Reed, T., Busnel, M. C., Marchaland, C., Roubertoux, P. L., et al. (1996). Comparative diagnoses of twin zygosity by SSLP variant analysis, questionnaire and dermatoglyphic analysis. Behavior Genetics, 26, 55-63.

Sumathipala, A., de Silva, N., Siribaddana, S. H., Abeysingha,M. R. N., \& Fernando, D. J. S. (2000).
Cross-cultural adaptation and preliminary validation of a zygosity determination questionnaire for twins in Sri Lanka. Twin Research, 3, 205-212.

Torgersen, S. (1979). The determination of twin zygosity by means of a mailed questionnaire. Acta Geneticae Medicae et Gemellologiae, 28, 225-236.

\section{Appendix A}

\section{Zygosity Questionnaire for Twins' Mothers}

I. Below you will find 16 questions on how alike your twin children were when "about one year of age”. Please mark off one answer for each item.

1 facial appearance

the number of the hair whorl

the position of the hair whorl

shape of eyebrow

shape of eyelid

shape of eye

shape of ear

voice

the number of the mole or spot

the position of the mole or spot

shape of fingers

physique

sleeping face

sleeping posture

liability to get illness

tendency of concordance for illness

No difference
1
1
1
1
1
1
1
1
1
1
1
1
1
1
1
1

$\begin{array}{cc}\text { Do not know } & \text { Clear difference } \\ 2 & 3 \\ 2 & 3 \\ 2 & 3 \\ 2 & 3 \\ 2 & 3 \\ 2 & 3 \\ 2 & 3 \\ 2 & 3 \\ 2 & 3 \\ 2 & 3 \\ 2 & 3 \\ 2 & 3 \\ 2 & 3 \\ 2 & 3 \\ 2 & 3 \\ 2 & 3\end{array}$

II. Below you will find three questions on how alike your twin children were when "about one year of age". Please mark off one answer for each item.

17 Were your twin children "as alike as two peas in a pod?"

$1 \quad$ As alike as two peas in a pod

2 Usual sibling similarity

3 Quite different

18 Were they mixed up at that age?

1 Yes, very often

2 Now and then

3 Never

19 By whom were they mixed up?

1 Parents

2 Relatives or neighbors

3 Others

4 Nobody 


\section{Appendix B}

\section{Zygosity Questionnaire for Twins}

Below you will find three questions about how alike you and your twin were in childhood. Please mark off the answers which are most fitting.

1 Were you and your twin "as alike as two peas in a pod?"

1 As alike as two peas in a pod

2 Usual sibling similarity

3 Quite different

$2 \quad$ Were you and your twin mixed up as children?

1 Yes, very often

2 Now and then

3 Never

3 In that case, by whom were you mixed up?
1 Parents
2 Teachers
3 Others
4 Nobody

\section{Appendix C}

\section{Definition of Three Similarity Indexes}

\begin{tabular}{lcc} 
Answer & Mothers of $M Z$ & Mothers of $D Z$ \\
\hline No difference & $a$ & $b$ \\
Do not know & $c$ & $d$ \\
Clear difference & $e$ & $f$ \\
\hline$a+b+c+d+e+f=t$. & \\
Index $A=(a+f) / t \times 100 . \operatorname{lndex~} B=(b+e) / t \times 100$. Index $C=(c+d) / t \times 100$ \\
Index $A+$ Index $B+$ Index $C=100$.
\end{tabular}

